\title{
Vorwort der Herausgeber/innen
}

\author{
Falko von Ameln ${ }^{1}$ Telse Iwers-Stelljes ${ }^{2} \cdot$ Simone Kauffeld $^{3}$ Karin Lackner ${ }^{4}$
}

Online publiziert: 18. März 2016

(C) Springer Fachmedien Wiesbaden 2016

2016 markiert den Beginn einer neuen Fachzeitschrift: „Gruppe. Interaktion. Organisation. Zeitschrift für Angewandte Organisationspsychologie (GIO)“. Mit der vorliegenden Ausgabe 1/2016 stellt sich die neue Zeitschrift GIO den Leser/-innen und Autor/-innen mit ihrem wissenschaftlichen und praxisorientierten Anspruch vor. Die Zeitschrift richtet sich dabei einerseits an wissenschaftlich orientierte Leser/innen aus der Organisationspsychologie und angrenzenden Wissenschaftsbereichen, andererseits an interne und externe Berater/innen, Supervisor/innen, Coaches und Führungskräfte.

Die Zeitschrift GIO geht aus der Zeitschrift „Gruppendynamik und Organisationsberatung " hervor, welche 46 Jahrgänge lang das Publikationsforum für gruppendynamische Forschung und Praxis war.

Priv.-Doz. Dr. F. von Ameln

von.ameln@gmx.de

1 Am Diekschloot 11b, 26506 Norden, Deutschland

2 Fakultät für Erziehungswissenschaft, Universität Hamburg, Hamburg, Deutschland

3 Arbeits-, Organisations- und Sozialpsychologie, TU Braunschweig, Braunschweig, Deutschland

4 Organisationsberatung, Supervision und Coaching, Universität Kassel,

Kassel, Deutschland

\section{Das Feld: Organisationspsychologie und interdisziplinäre Perspektiven}

Hinter dem Akronym GIO stehen drei Begriffe: Gruppe. Interaktion. Organisation. Soziale Systeme und ihre Dynamik sowie die in diesen Systemen stattfindenden Interaktionen können nicht eindeutig einer traditionellen wissenschaftlichen Disziplin zugeordnet werden. Gerade die Organisation ist Gegenstand verschiedener Fachrichtungen: Sie kann aus einer betriebswirtschaftlichen, einer soziologischen, einer psychologischen, einer pädagogischen oder einer philosophischen Perspektive betrachtet werden. Entsprechend der disziplinübergreifenden Natur ihres Gegenstandes lotet die GIO, ausgehend von einer klaren Verortung in der Organisationspsychologie, diese unterschiedlichen Perspektiven aus.

\section{Das Ziel: Erkenntnisse an der Schnittstelle von Wissenschaft und Praxis}

Die Organisationspsychologie ist eine von Natur aus angewandte Wissenschaft. Dementsprechend wollen wir aus der Befruchtung von Wissenschaft und Praxis relevante Erkenntnisse sowohl für wissenschaftliche Leser/innen als auch für Praktiker/innen gewinnen. Dabei wollen wir nicht nur der Tradition der Gruppen- und Organisationsdynamik verpflichtet bleiben, sondern vor allem den aktuellen Entwicklungen der Organisationspsychologie in all ihren Facetten gerecht werden und das Spektrum sozialwissenschaftlicher Wissenschaft und Praxis erweitern. 


\section{Die Texte: Forschung und Anwendung im Dialog}

Theoriebeiträge werden ausführlich über den aktuellen Forschungsstand eines Themas informieren - in diese Kategorie fallen wissenschaftliche Studien, Feldforschungsprojekte, Sozialexperimente zu relevanten und verwandten Themen und theoretische Analysen von Fallbeispielen. Die wissenschaftstheoretische Fundierung der Beiträge kann sowohl klassisch-empirischen als auch partizipativen Forschungsdesigns folgen. So ist es einerseits der Erkenntnisgewinn aus einem Forschungsvorhaben, der GIO für die Leser/-innen attraktiv macht, andererseits können theoretisch reflektierte Fallbeispiele bestechen und zu weiteren Forschungsvorhaben anregen. Die Forschungslandschaft der Sozialwissenschaften ist und wird zunehmend interund transdisziplinär. Die neue Zeitschrift GIO greift die Tradition der Participatory Action Research auf und widmet sich in einzelnen Beiträgen der wissenschaftstheoretischen Entwicklung dieser relativ jungen Forschungsphilosophie.

Dem Grundgedanken der Zeitschrift folgend, sind Forschungsergebnisse aller Art auf deren Anwendung in der Praxis zu überprüfen. Auf die Frage: „Was können Praktiker/innen aus der Studie lernen?" sind Autor/innen der GIO aufgefordert, Antworten zu geben.

Bei den Praxisbeispielen sind es vor allem die Interventionen, die den Leser/innen neue Handlungsmöglichkeiten aufzeigen sollen. Erkenntnisgewinn aus Praxisbeispielen gewinnen die Leser/innen u. a. durch theoretische Auseinandersetzungen mit dem Praxisbeispiel.

\section{Tradition und Zukunft: Von der Gruppendynamik zur Participatory Action Research}

Über vierzig Jahre lang war die „Gruppendynamik“ - wie sie umgangssprachlich bezeichnet wurde - eines der bedeutendsten Foren für die wissenschaftliche und praktische Auseinandersetzung mit einer inter- und transdisziplinären Fachrichtung, deren Anfänge in den 40iger Jahren des vorigen Jahrhunderts liegen. Kurt Lewin hat als einer der Begründer gruppendynamischer Konzeptionen mit seinen Erkenntnissen die Welt der Wissenschaft bereichert. Dieser historischen Wurzel entsprechend wurde das letzte Themenheft der Zeitschrift für Gruppendynamik und Organisationsberatung seinen Spuren gewidmet.

Die Entwicklungen der Wissenschaft, und hier vor allem der Sozialwissenschaft, bestätigen den Lewin'schen Gedankengang. Die von Lewin begründete Aktionsforschung hat sich weiterentwickelt. Im nordamerikanischen Raum spricht man von „Participatory Action Research“, in Deutschland von „Partizipationsforschung“, in Öster- reich von „Interventionsforschung“. Allen gemeinsam ist der Gedanke, die beforschten Personen und Personengruppen in den Forschungsprozess miteinzubeziehen, sie zu aktiven Gestalter/innen eines Forschungsvorhabens zu machen, in dem diese selbst auch handelnde Akteur/innen sind. Dieser Grundgedanke findet sich gegenwärtig im Konzept der sozialen Nachhaltigkeit wieder. Partizipation, die aktive Beteiligung der betroffenen Personen an Entscheidungsprozessen, setzt ,aufgeklärte“ Teilnehmer/innen und Systeme voraus. Hier berührt die gruppendynamische Tradition pädagogisches Terrain. Die gruppendynamische Tradition erhebt den Anspruch, im Sinne einer aufgeklärten Gesellschaft politisch bildend zu sein. Die Zeitschrift GIO ist diesem Grundgedanken weiterhin verpflichtet, indem sie aktuelle gesellschaftspolitische Themen im organisationalen Kontext aufgreift und diese aus einer wissenschaftlichen Perspektive betrachtet.

Die verschiedenen Forschungsstränge zu bündeln und deren Transformation in praktische Relevanz zu erörtern, ist Anliegen der neuen GIO und Verpflichtung ihrer Akteure.

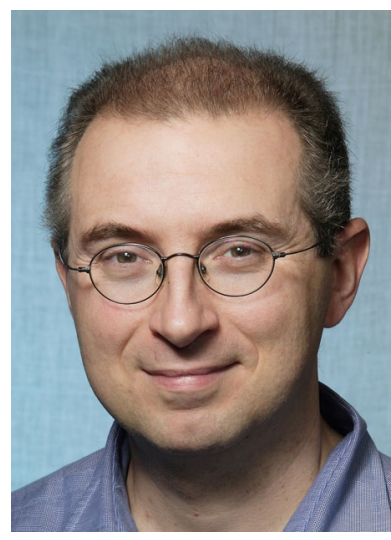

Priv.-Doz. Dr. Falko von Ameln, Psychologe, Habilitation im Fach Beratungswissenschaft an der Alpen-Adria-Universität Klagenfurt, Aus- und Weiterbildung von Berater/innen an verschiedenen Universitäten und privatwirtschaftlichen Instituten. Forschungs- und $\mathrm{Pu}-$ blikationsschwerpunkte: Theorie und Methodik der Organisationsberatung, Systemtheorie, Führung, Macht, Psychodrama.



Prof. Dr. MHEd. Telse IwersStelljes, Erziehungswissenschaftlerin Gestalttherapeutin und $\mathrm{Su}$ pervisorin. Universität Hamburg, Fakultät für Erziehungswissenschaft. Forschungsschwerpunkte: Führungs- und Aufstiegskompetenz von Frauen; Professionalisierung durch Beratung und Coaching im Lehramt; mentale Blockaden und Prozesse deren Lösung durch Selbstregulation; Entwicklung von Achtsamkeit durch Introvision. 


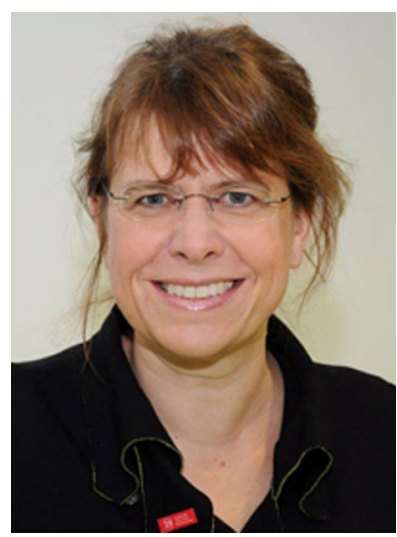

Prof. Dr. Simone Kauffeld, Lehrstuhl für Arbeits-, Organisationsund Sozialpsychologie. TU Braunschweig. Forschungsschwerpunkte: Teaminteraktionen, Kompetenzdiagnose, -entwicklung und -management, Karriereentwicklung und Coaching, Führung und Veränderung.

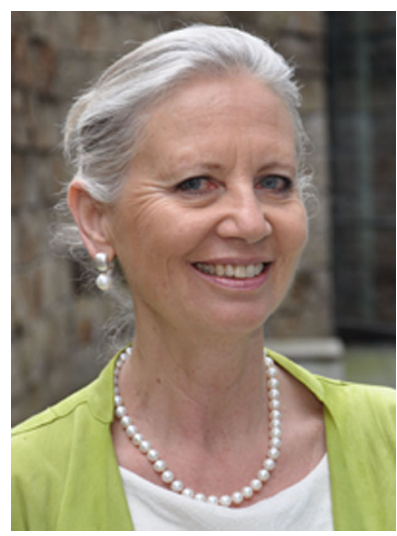

Prof. Dr. Karin Lackner, Lehrstuhl für Organisationsberatung, Supervision und Coaching, Universität Kassel. Forschungsschwerpunkte in den Feldern Beratung, Organisation, Gruppendynamik. Participatory Action Research, transdisziplinäre Forschung. Masterstudiengang MDO (Mehrdimensionale Organisationsberatung). 